\title{
Is general anaesthesia still the easiest option?
}

\author{
Stephen Hancocks OBE \\ Editor-in-Chief \\ The BDJ Upfront section includes editorials, letters, news, book reviews and interviews. Please direct your correspondence to the News Editor, \\ Kate Quinlan at BDJNews@nature.com. Press releases or articles may be edited, and should include a colour photograph if possible.
}

$\mathrm{D}$ ental general anaesthesia (DGA) for adults is one of those activities which for the most part is 'done somewhere else'. If we're honest it can prove to be a bit of a headache, a bit of a nuisance. To whom to refer the patient? While the topic in relation to child dental health has been the subject of much exposure in the media recently due to the continuing high levels of caries in some children, in adults it remains a slightly shady, un-discussed matter, something that somebody else does.

The authors of a paper published in this issue, Hong and Baker, wish to encourage professional debate about this area and have undertaken a survey to add some data to their cause. ${ }^{1}$ They explored the current perceptions and opinions of clinicians in oral and maxillofacial surgery units throughout the UK on various aspects of DGA and uncovered some insightful views.

I suspect it might come as a surprise to more recent graduates that general anaesthesia (GA) was previously allowed in general dental practice, but was banned from January 2002 following a series of deaths, mainly of children, which drew considerable coverage in the media. The then Chief Medical Officer, launching a report entitled $A$ conscious decision, ${ }^{2}$ which itself followed from a widely debated report in 1990, dubbed the Poswillo report after the name of the leading author, announced that henceforth all GAs would have to take place in a hospital. ${ }^{3}$ Immediately prior to the ban some 57,000 GAs were performed each year in practices, down from 300,000 in the mid-1990s and over a million in the mid-1970s, credited mainly to the rising use of sedation techniques.

Dental anxiety is familiar to all of us; a fact of life with which we learn to cope both professionally and socially. How often at the time when we are introduced do we face the 'oh, I hate dentists' comment? This is not to undermine the seriousness of the condition or deny that it does have a great impact on the lives of those who suffer particularly at the extreme end of the spectrum. However, is a DGA still a knee-jerk reaction, an easy option? What if the patient requests it, insists on it?

For some patients, such as those with learning difficulties, the need is clearly apparent and a DGA is the only realistic option. For others though the choices are treatment with of pain, then yes, arguably but what of the medium- to longer-term prospects? Will this patient continue to require a GA every time in the future? Wouldn't it be kinder to try and help them into accepting at least sedation or possibly care while conscious, treating the condition rather than merely the symptoms.

In an ideal world the answer must be yes but the point that the authors effectively raise in their paper is that we do not live in such a utopia. So what do we do as a profession and as a society? Do we shrug and carry

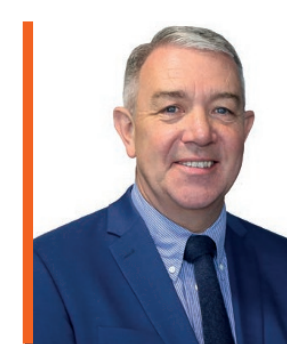

'Is a dental general anaesthetic still a knee-jerk reaction, an easy option?' local anaesthetic or by using some form of sedation. There are proven methods to pave the way for the former through psychological pathways, one of which that has emerged of recent times being cognitive behaviour therapy. However, the opening gambit in describing the technique suggests that anything up to ten sessions might be needed in order to make progress. The problem of resource is immediately evident, especially in an NHS already stretched on so many fronts. It is not surprising that a lay opinion may well be that surely a single appointment GA is a much swifter, cheaper and kinder alternative. But is it?

Swifter; certainly if the patient is in pain and the prospect of multiple counselling sessions lays ahead. Cheaper; certainly not given that a whole operating theatre with anaesthetist is required compared to a dental surgery and a local anaesthetic cartridge. Kinder? More difficult to assess. For getting the patient out on or do we attempt to tackle some of the underlyining causes these many years since the rules changed?

DGA lurks under the edges of the normal-distribution graph rather as does jam trapped below the rim of a jar; frustratingly difficult to access with spoon, knife or any other instrument and yet impossible to ignore. Do we continue to reach for the GA consent form in the same way that we pop the jam jar in the dishwasher and hope it doesn't happen again in the future, or do we start to look for a better way?

DOI: 10.1038/sj.bdj.2017.143

1. Hong B, Baker A. General anaesthetic service for adult dental extractions: an 'À La Carte Menu'? Survey results. Br Dent J 2017; 222: 261-267.

2. A conscious decision, a review of the use of general anaesthesia and conscious sedation in primary dental care. London: Department of Health, 2000.

3. General anaesthesia, sedation and resuscitation in dentistry: report of an expert working party. London: Department of Health, 1990. 